in $27.6 \%$ of cases. Overexpression correlated closely with decreased overall survival in this population (univariate hazard ratio 2.59; $P=0.004)$; this effect was partly confounded by International Federation of Gynecology and Obstetrics (FIGO) stage in multivariate analysis. Median overall survival was 40.3 months in patients who overexpressed HER2, and greater than 168.7 months in those with low HER2 expression.

In breast cancer, HER2 overexpression affects survival by increasing the potential for tumor metastasis - an effect mediated by the chemokine-chemokine-receptor pair SDF-1/ CXCR4 complex. Pils et al. hypothesized that pathways of oncogenesis could be similar in breast and ovarian cancers and, therefore, examined whether the SDF-1/CXCR4 system was involved in the process leading to compromised survival in ovarian cancer. Cytoplasmic CXCR4 expression correlated with membranous SDF- 1 abundance $(P<0.001)$, but neither of these potential oncoproteins correlated with HER2 overexpression, nor did they predict overall survival. These results indicate that the poorer survival associated with HER2 overexpression in ovarian cancer was independent of the SDF-1/CXCR4 system. Consequently, HER2 downstream processes probably differ between breast and ovarian cancers.

Original article Pils D et al. (2007) In ovarian cancer the prognostic influence of $H E R 2 / n e u$ is not dependent on the CXCR4/SDF-1 signalling pathway. Br J Cancer 96: 485-491

\section{Neoadjuvant chemotherapy improves chance of optimum debulking in ovarian carcinoma}

Achievement of optimum cytoreduction following initial debulking of epithelial ovarian cancer (EOC) has been shown to be a favorable prognostic factor, but optimum debulking is achieved in only $50-60 \%$ of women presenting with advanced EOC. Evidence is accumulating to indicate that chemotherapy before surgery, termed neoadjuvant chemotherapy (NAC), might increase the proportion of women for whom optimum debulking is possible.

In a retrospective review of 172 patients with advanced EOC, Hou et al. compared survival in patients who underwent primary debulking surgery (PDS) followed by adjuvant platinumbased chemotherapy $(n=109)$, with survival in those who received NAC plus debulking $(n=63)$. Among patients in the NAC group, 37 received carboplatin plus paclitaxel and 26 received carboplatin plus cyclophosphamide. Patients who underwent NAC had a significantly shorter operating time and hospital stay than patients who underwent PDS $(P<0.0001$ for both). Optimum debulking was achieved in $95 \%$ of NAC patients compared with $71 \%$ of patients who underwent PDS $(P<0.001)$. Among the patients in the NAC group, those who received carboplatin plus paclitaxel had a significantly longer median overall survival than those who received carboplatin plus cyclophosphamide (83 months vs 26 months; $P=0.008$ ). Patients with extraabdominal disease who received NAC with carboplatin plus paclitaxel had a significantly longer median overall survival than those with extra-abdominal disease who received PDS (31 months vs 20 months; $P=0.032$ ).

Most studies of NAC have involved only women unsuitable for PDS; however, on the basis of these results, the authors conclude that NAC could have a wider role in the management of advanced EOC.

Original article Hou JY et al. (2007) Neoadjuvant chemotherapy lessens surgical morbidity in advanced ovarian cancer and leads to improved survival in stage IV disease. Gynecol Oncol 105: 211-217

\section{MUC4 expression might predict outcome in patients with lung adenocarcinoma}

The tracheobronchial mucin MUC4 is a ligand for the ErbB2 tyrosine kinase, and influences expression of p27, a cyclin-dependent kinase inhibitor that regulates the $\mathrm{G} 1$ and $S$ phases of the cell cycle. Previous studies have shown that MUC4 expression is a prognostic factor in invasive ductal carcinoma of the pancreas and intrahepatic cholangiocarcinoma. Tsutsumida et al. have now shown that MUC4 expression also correlates with prognosis in small-sized lung adenocarcinomas.

Surgically resected specimens (less than $3 \mathrm{~cm}$ ) from 185 patients with nonbronchioloalveolar type lung adenocarcinoma were assessed. Immunohistochemical analysis revealed that MUC4 was expressed in both the cytoplasm and membrane of carcinoma cells, with cytoplasmic expression being more 